International Journal on Cybernetics \& Informatics (IJCI) Vol. 5, No. 4, August 2016

\title{
Blind IMAge Quality Assessment With Local CONTRAST FEATURES
}

\author{
Ganta Kasi Vaibhav, \\ PG Scholar, Department of Electronics and Communication Engineering, \\ University College of Engineering Vizianagaram,JNTUK. \\ Ch.Srinivasa Rao, \\ Professor, Department of Electronics and Communication Engineering, \\ University College of Engineering Vizianagaram,JNTUK.
}

\begin{abstract}
The aim of this research is to create a tool to evaluate distortion in images without the information about original image. Work is to extract the statistical information of the edges and boundaries in the image and to study the correlation between the extracted features. Change in the structural information like shape and amount of edges of the image derives quality prediction of the image. Local contrast features are effectively detected from the responses of Gradient Magnitude $(G)$ and Laplacian of Gaussian $(L)$ operations. Using the joint adaptive normalisation, $G$ and $L$ are normalised. Normalised values are quantized into $M$ and $N$ levels respectively. For these quantised $M$ levels of $G$ and $N$ levels of $L$, Probability $(P)$ and conditional probability $(C)$ are calculated. Four sets of values namely marginal distributions of gradient magnitude $P g$, marginal distributions of Laplacian of Gaussian Pl, conditional probability of gradient magnitude Cg and probability of Laplacian of Gaussian Cl are formed. These four segments or models are Pg, $\mathrm{Pl}, \mathrm{Cg}$ and $\mathrm{Cl}$. The assumption is that the dependencies between features of gradient magnitude and Laplacian of Gaussian can formulate the level of distortion in the image. To find out them, Spearman and Pearson correlations between $\mathrm{Pg}, \mathrm{Pl}$ and $\mathrm{Cg}, \mathrm{Cl}$ are calculated. Four different correlation values of each image are the area of interest. Results are also compared with classical tool Structural Similarity Index Measure (SSIM)
\end{abstract}

\section{KEYWORDS}

Gradient Magnitude, Laplacian of Gaussian, Joint Adaptive Normalisation, Normalised Bivariate Histograms, Spearman rank Correlation, Pearson Correlation Coefficient.

\section{INTRODUCTION}

Image quality assessment evaluates the quality of the distorted image. Factors which determine image quality are, noise, dynamic range tone reproduction, colour accuracy, distortion, contrast, exposure accuracy, lateral chromatic aberration, sharpness, colour moiré, vignette, artefacts. Distortion is defined as abnormality, irregularity or variation caused in an image. This is noticeable in low cost cameras. Distortions are caused during Acquisition, Compression, Transmission and Storage. Changes in image or quality of image are observed either by the human subjects called as subjective measure or calculated by mathematical operations called as

DOI: $10.5121 /$ ijci.2016.5422 
International Journal on Cybernetics \& Informatics (IJCI) Vol. 5, No. 4, August 2016

objective measures. Image quality assessment can also be categorised as With Reference models and Without Reference models. First type of models finds out quality of the image by comparing with its original image. Second type of models also called as Blind image quality assessment finds out the quality of distorted image without comparing with its original image.

Local contrast features describe the structure of the image. The changes in the structure of the image like shape and amount of edges are detected easily. Two general local contrast features are Gradient magnitude and Laplacian of Gaussian. Joint adaptive normalisation (JAN) normalises G and $\mathrm{L}$ channels jointly. The benefit of JAN is to make the horizontal, vertical and diagonal features correlative in the image. It reduces the redundancies in image. Normalisation stabilizes the profiles of these features. The $\mathrm{G}$ and $\mathrm{L}$ distributions of images are very different from natural images. Quantising into levels and thereby giving joint probability function, statistics are derived. Marginal distributions and conditional probability dependency measures are recorded into four different correlations are drawn.

Over years there had been several performance measures for image quality assessment. DMOS/MOS difference mean opinion scores is a subjective measure which evaluates over human judgements [4]. Various databases established by IQA community are LIVE, CSIQ, TID2008. CSIQ, TID2008 and LIVE has 4 common types of distortions they are JP2K, JPEG, WN and Gaussian blur. These are used to identify the characteristics of the various distortions. To find out performance of a method, a machine which calculates the correlations of the subjective scores of the human judgements constructed. The correlations used are Spearman rank order correlation coefficient (SRC) and Pearson correlation coefficient (PCC). Proposed Blind image quality assessment model is compared with Structural Similarity Index Measure (SSIM).

Experimental results define that there is equivalent information in one of the four sets of statistics we derive. However joint statistics in of Pearson model give better results. Existing models involve in large procedures to find out the disturbances in the frequencies over different distortions. Few models even changes the features of the image. $\mathrm{G}$ and $\mathrm{L}$ operations are close to the results of the human visual system. G and L are independent over the distortions. The marginal and independency distributions can determine the quality of the image. Joint adaptive normalisation procedure normalises the $\mathrm{G}$ and $\mathrm{L}$ features. Proposed model uses independency distributions to measure joint statistics. Which leads to highly competitive results in terms of Quality prediction, Generalisation ability, and effectiveness. Existing models have computational complexity. To reduce regression methods, and get finer quality measure with no training or regression methods we derive a new method with probability statistics.

This paper is organised in four sections. Section II gives a brief study of all the existing models of without reference image quality assessment. Section III presents the features of the proposed model in detail and exclusive experimental results in each stage of the process. Section IV concludes the paper.

\section{RELATED WORK}

\subsection{Literature Survey}

There are several image quality assessment models. Mean Square Error (MSE) is the primitive measure [2]. When original image is the known reference image, a written explanation of the measure exits in the literature. Mean Square Error (MSE) found to be very important measure to 
compare two signals. It provides a similarity index score that gives the degree of similarity. Similarity index map is amount of distortion between two signals. It is simple, parameter free and inexpensive. It is employed widely for optimizing and assessing signal processing applications. Yet it did not measure signal fidelity to certain required extinct. When altered by two different distortions at a same level, MSE only gave the value of distortion. MSE is very converse to the human perception. MSE led to development of Minimum Mean Square Error, Peak Signal to Noise Ratio.

Based on the luminance, contrast and structure of an image, the Structural Similarity Index Measure (SSIM) [3] is developed. It is an objective quality measure of image to quantify the visibility of errors. It is a similarity measure for comparing any two signals. Like MSE, it is a full reference model which depends on the structural similarity. It has SSIM index map which shows the comparison better than MSE. It is a complex measure for an image with large content. Though SSIM index could give better results compared to the traditional Mean square error, it lacked in defining what the type of distortion is present in the image. This novel work gave scope for the study on structure statistics of the image. Furthermore, it is used to simplify algorithms of image processing system. For image quality measure, SSIM proved its efficiency than mean square error. SSIM is computationally expensive than MSE.

Difference Mean Opinion Scoring (DMOS) is a performance evaluation study of existing methods of IQA along with subjective scores collected over a period of time with numerous human subjects [4]. There is no replacement to the Human Visual system (HVS) [5]. DMOS when compared objective measures like PSNR, MSE and SSIM, proved that Human Visual System had better evaluation. Subjective group verified and gave responses over LIVE database which consists of 779 distorted images from 29 original images with five distortions [14]. It gave importance to visual difference. Used of Spearman Rank Correlation and Root Mean Square Error for perfection. It has $95 \%$ confidence criterion of finding out whether distorted or not. This study gave a valuable resource of scores of distortion. It led to study of natural scene statistics. DMOS is used as benchmark for checking of constructed models.

The reference image is not always available. Hence, there is need of without reference image quality assessment measure also called as Blind Image Quality Assessment. It assesses the quality of an image completely blind i.e., without any knowledge of source distortion [5]. Distorted Image Statistics (DIS) which is used to classify images into distortion categories gave the ease to decide type of the distortion [5]. In this literature, wavelet transform is performed on the image indices. Shape parameter is defined using Gaussian distribution. Given a training set and testing setoff distorted images, a classifier Support Vector Machine is used to classify image into five distortions. This is the first without reference method. It could differentiate the type of distortion. Results of this model correlates with reference models. Its drawback is its computational complexity. This methodology can be replaced with any module which performs better i.e. either by increasing the number of distortions or by increasing training set for better results. This model can be used for video processing by adding measure of relevant perceptual features.

With the probability of usage of the indices as features, a new approach BLIINDS is proposed [5]. It is a model with the evolution of features derived from the Discrete Cosine Transform domain statistics. While the previous no reference is distorted specific approaches, this approach could explain the type of the distortion. It used Support Vector Machine (SVM) which correlates well with human visual perception. It is computationally convenient as it is based on a DCTframework entirely, and beats the performance of Peak signal to noise ratio. The probabilistic prediction model was trained on a small sample of the data, and only required the computation of 
International Journal on Cybernetics \& Informatics (IJCI) Vol. 5, No. 4, August 2016

the mean and the covariance of the training data. It computes blockiness measure. It estimates the particular type of distortion. Time taken for computation is high.

Final evolution is the reduction of complexity by combination with Natural scene statistics and addition of distorted image statistics. Work is to extract the statistical information of the edges and boundaries in the image and to study the correlation between the extracted features. Change in the structural information like shape and amount of edges of the image derives quality prediction of the image. Local contrast features are effectively detected from the responses of Gradient Magnitude (G) and Laplacian of Gaussian (L) operations. Adaptive procedures are used to normalize the values of $\mathrm{G}$ and $\mathrm{L}$. Normalized values are quantized into certain levels respectively. Conditional probability and Marginal distribution of $\mathrm{G}$ and $\mathrm{L}$ are calculated which are stored into three segments. They proposed three models. These three segments or models are M1, M2, M3 which have only conditional probability values, only marginal distribution values and both conditional probability and marginal distribution values. Loading values in the support vector regression; over the set of images collected from LIVE database a probable score is determined for each distortion.

There is complexity in training these values into the support vector machine. This paper is a thorough study of the conditional probability and marginal probability values of the gradient magnitude and Laplacian of Gaussian. Marginal distributions and conditional probabilities and their dependencies which lead to highly competitive performance are employed in this work. This avoided the training and learning of the features. Therefore, the complexity is reduced in the modelling. This procedure is a direct extraction of the amount of edges and change in the image. Intensive measurement of the structural information is derived from the correlations between the amounts of the variation caused in the image. Four correlations namely Pearson correlation coefficient between $\mathrm{Pg}$ and $\mathrm{Pl}$ (PRCP), Pearson correlation coefficient between $\mathrm{Cg}$ and $\mathrm{Cl}$ (PRCQ), Spearman rank correlation between Pg and Pl (SRCP), Spearman rank correlation between $\mathrm{Cg}$ and $\mathrm{Cl}$ (SRCQ) are proposed in this paper.

\section{PROPOSED WORK}

As discussed in the above section, a methodology to find out the profiles of the structural features and to derive the correlations between the structural features is explained in stages with their relevant outcome in each stage is given below.

\subsection{Local Features}

Local contrast features give the information of the amount of change in the structure of an image. Two general local contrast features are Gradient magnitude (G) and Laplacian of Gaussian (L).

Discontinuities in the structural details like luminance of an image or the change in the intensities are important for quality assessment. These can be derived by performing gradient magnitude and Laplacian of Gaussian operations. We use CSIQ database which is commonly used international database for image quality assessment. Trolley image is considered from database and denoted by I. To standardize Convert image from RGB to grayscale. For size of the image, in the following stages gradient magnitude and Laplacian of Gaussian operators are applied. 


\subsection{Gradient Magnitude}

Gradient magnitude is the first order derivative, often used to detect the edges in the image. Expression for gradient magnitude is given as:

$$
G=\sqrt{[I * V]^{2}+[I * H]^{2}}
$$

The vertical prewitt filter kernel (V) is considered as $\left[\begin{array}{lllllllll}-1 & -1 & -1 ; & 0 & 0 & 0 ; & 1 & 1 & 1\end{array}\right]$. The horizontal

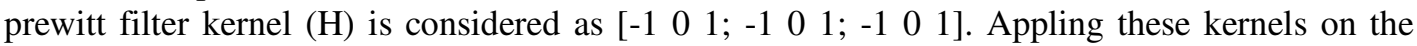
image and substituting in $\mathrm{G}$ expression given above, we get gradient magnitude image. The original image, vertical prewitt filtered image, horizontal prewitt filtered image and the resultant gradient magnitude image are shown in figure 1.

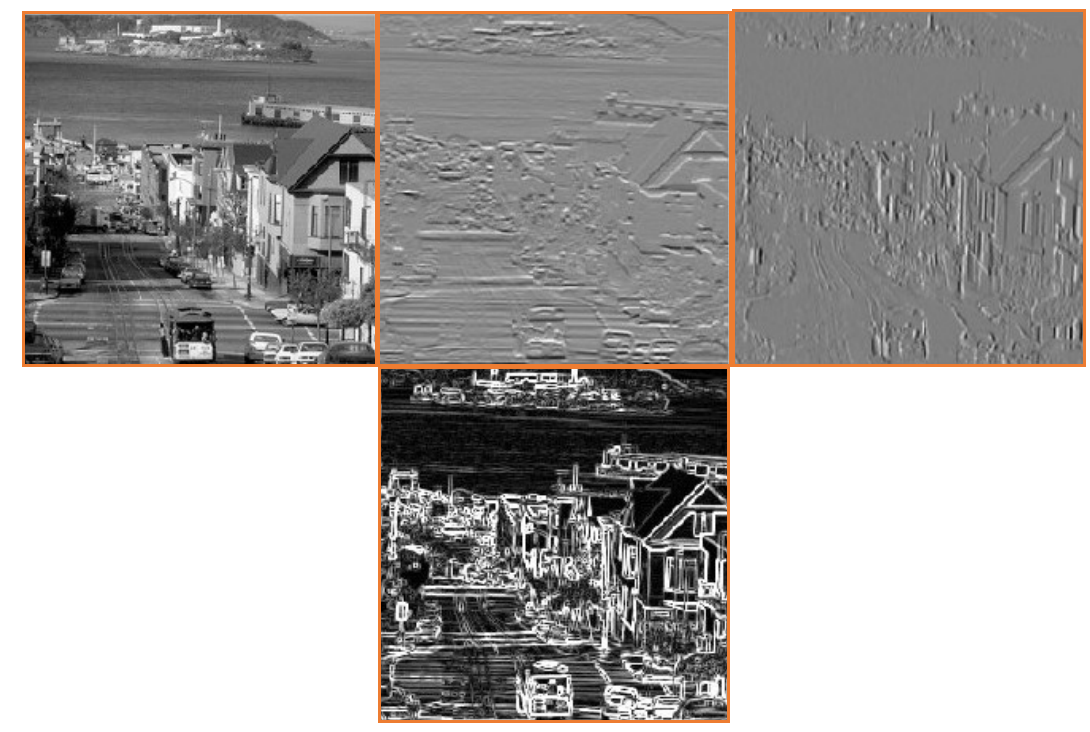

Figure.1. 1) Original image 2) Vertical Prewitt Filtered Image 3) Horizontal Prewitt Filtered Image 4) Resultant gradient magnitude image.

\subsection{Laplacian of Gaussian}

Laplacian of Gaussian is the second order derivative as shown in the equation. Expression of the Laplacian of Gaussian is given as,

$$
L=I * h_{L O G}
$$

Where,

$$
h_{\mathrm{L} a g}=\frac{\partial^{2}}{\partial x^{2}} g(x, y)+\frac{\partial^{2}}{\partial y^{2}} g(x, y)
$$

$\mathrm{G}$ and $\mathrm{L}$ operations reduce the spatial redundancies in the image. The Laplacian of Gaussian applied image is shown in figure 2. Some consistencies between neighbouring structures still remain. So, to remove these we perform joint adaptive normalisation. 
International Journal on Cybernetics \& Informatics (IJCI) Vol. 5, No. 4, August 2016

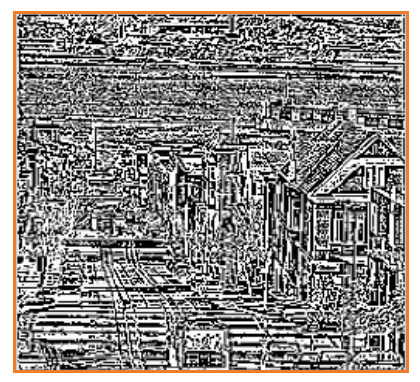

Figure 2. Laplacian of Gaussian performed image.

\subsection{Joint Adaptive Normalisation}

Joint adaptive normalization (JAN) is performed to remove the spatial redundancies remained in the image [1]. This decomposes the channel into different frequencies and orientations. According to the normalization factor $\mathrm{G}$ and $\mathrm{L}$ are reduced.

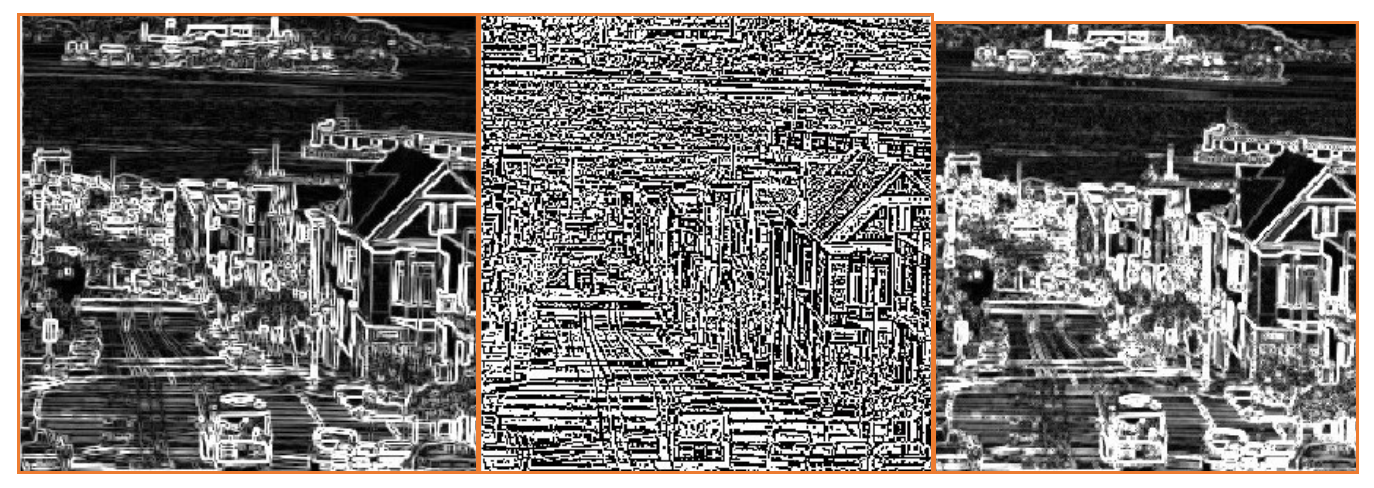

Figure 3. 1) Laplacian of Gaussian 2) Gradient Magnitude 3) Joint Factored Image

\subsection{Locally Adaptive Normalization Factor}

A $3 * 3$ mask which has values which when summated equals to 1 is applied on the image. As the mask is run over square of joint factored image while finding out the square root of the same, gives normalization factor. Last step of this procedure is to find out new values of $G(i, j)$ and $\mathrm{L}$ $(i, j)$ as $G^{\mid}(i, j)$ and $L^{\mid}(i, j)$ by reducing the features by normalisation factor. Variation in Buildings image before and after joint adaptive normalisation are shown in figure 4. 


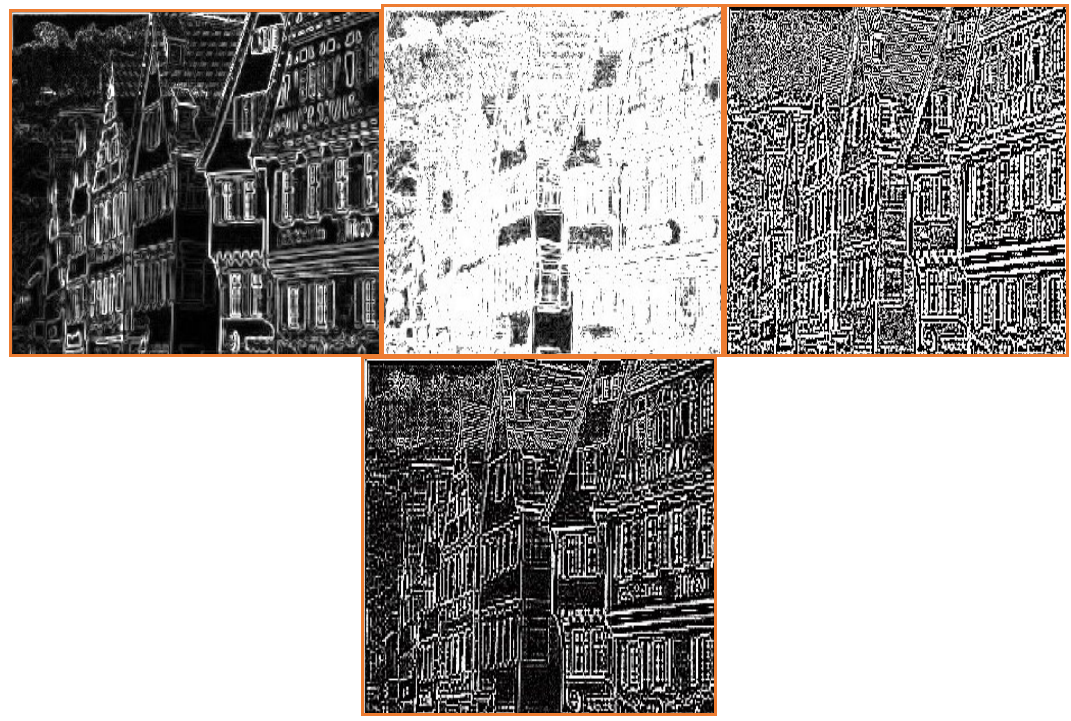

Figure 4. 1) Gradient Magnitude 2) GM after joint adaptive normalisation 3) Laplacian of Gaussian 4) Log after joint adaptive normalisation

\subsection{Quantization}

The features obtained on applying the techniques of gradient magnitude and Laplacian of Gaussian are quantised. This is performed to decrease the dynamic range and to bring the features into an optimum range. We quantized $G^{\mathrm{l}}(i, j)$ into planes as $\left\{G_{1}, G_{\eta}, \ldots G_{m}\right\}$. Similarly $L^{\mathrm{l}}(i, j)$ into $\left\{L_{1}, L_{2}, . . L_{n}\right\}$. In this case we take 17 levels of which we assign 17 different levels of pixels values. This may be a lossy process but it is done to derive the respective density functions of gradient magnitude and laplacian of Gaussian features. Resultant Trolley image after quantization into 17 levels is shown in figure 5.

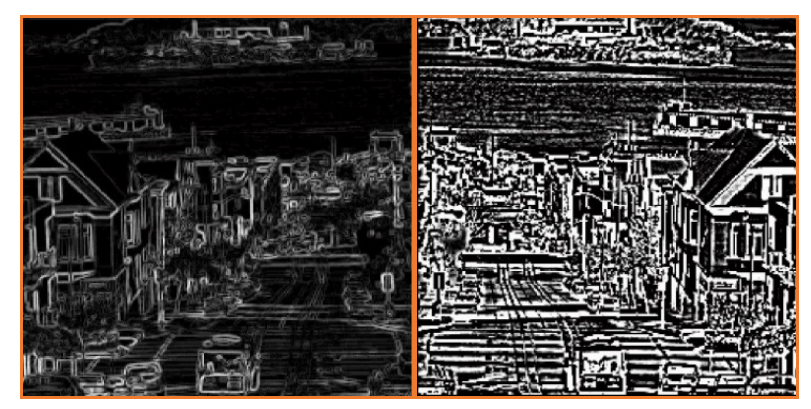

Figure 5. 1) GM quantised into 17 levels 2) LOG quantised into 17 levels

\subsection{Marginal Distributions and Conditional Probability}

Dependency measures like Marginal distributions and conditional probability closely relate the amount of distortion present in the image. This is more comprehensive evaluation of the extracted features. For the 17 levels of quantized images the marginal distributions and conditional 
probabilities are derived. To find out the marginal dependencies of $\xi^{\prime}$ and $L^{l}$, procedure starts with deriving the joint empirical functions for all levels.

$$
I E F_{m, n}=\left(G^{\mid}=G_{1}, G_{2}, \ldots G_{m}, L^{\mid}=L_{1}, L_{2}, \ldots L_{n}\right)
$$

Normalised histogram of $G^{\mid}$and $L^{\mid}$is $J F F_{n z, n}$. Marginal distributions of $G^{\mid}$and $L^{\mid}$are given in the expression below. Marginal probabilities of $G^{l}$ and $L^{\mid}$are shown in the figure 6 .

$$
\begin{gathered}
\operatorname{Pg}\left(G_{\mathrm{s}}^{\mid}=G_{m n}\right)=\sum_{\mathrm{r}=1}^{\mathrm{N}} J E^{\prime} F_{m, n} \\
\left.\operatorname{Pl}\left(L^{\mid}=L_{n}\right)\right)=\sum_{\mathrm{m}=1}^{\mathrm{M}} J E F_{m, n}
\end{gathered}
$$

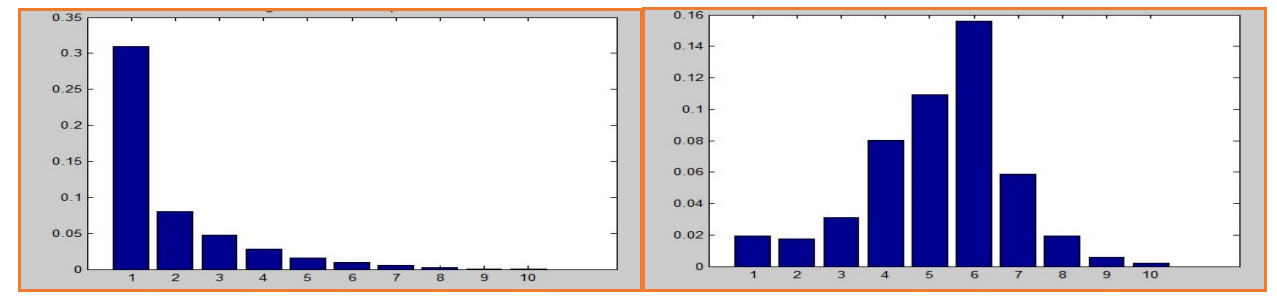

Figure 6. Marginal distributions of quantised $G^{\mid}$and $L^{\mid}$.

Sometimes the marginal distribution does not show the dependencies between $G^{\mid l}$ and $L^{l}$. The dependency between them are derived by dependency measure given in equation below.

$$
D E P_{m, n}=\frac{\rho E F_{\min }}{P(G \mid) * P(L)}
$$

Using the marginal distributions as the weights the conditional probabilities are derived for $G^{\text {l }}$ and $L^{\mid}$as $\mathrm{Cg}$ and $\mathrm{Cl}$. These probability distributions are otherwise called as the independency distributions. Independency distributions of $G^{l}$ and $L^{l}$ are shown in figure 7.

$$
\begin{aligned}
& \mathrm{Cg}\left(G^{\mathrm{l}}=G_{m}\right)=\operatorname{Pg}\left(G^{l}\right) \cdot \frac{1}{N} \sum_{n-1}^{\mathrm{N}} D E P_{m, n} \\
& \mathrm{Cl}\left(L^{\mid}=L_{z}\right)=\operatorname{Pl}\left(L^{\mid}\right) \cdot \frac{1}{M} \sum_{m=1}^{\mathrm{M}} D E P_{m, n}
\end{aligned}
$$

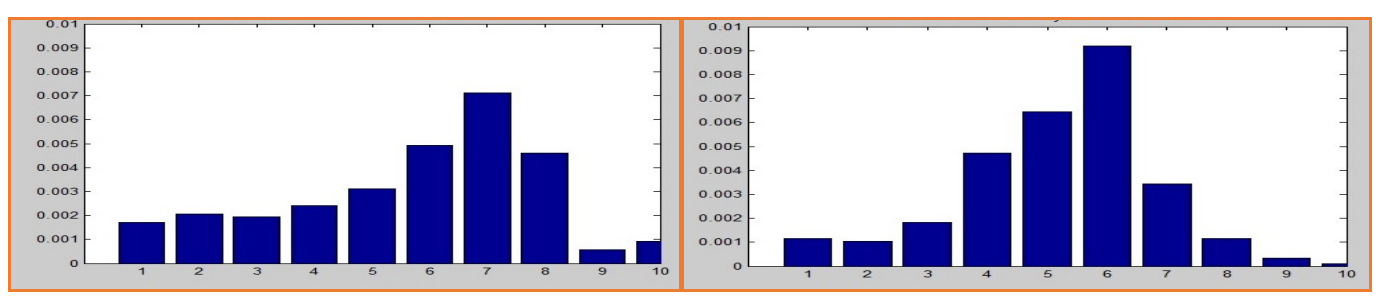

Figure 7. Independency distributions of $G^{\mathrm{I}}$ and $\boldsymbol{L}^{\mid}$. 
While finding out the marginal densities of GM and LOG and their corresponding profiles, it is seen that changes in a distorted image and randomness of distortion is distinguishable through.

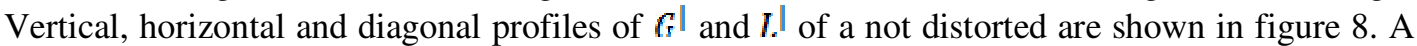
normal distortion less image has quite different distributions from that of a distorted version of it. Similarly their profiles are also plotted. The individual random process of $\mathrm{G}$ and $\mathrm{L}$ features after quantization are assumed as the random variables. The below figures fall under the assumption of binomial distribution of the data. Further there is need to identify the dependency between the distributions of $\mathrm{G}$ and $\mathrm{L}$. Hence, calculating joint probability density function between them is the solution. In a general case they find to be independent and results are in product of their individual marginal density functions. For sample profiles over 20 bins are shown in plots in figure 8 .

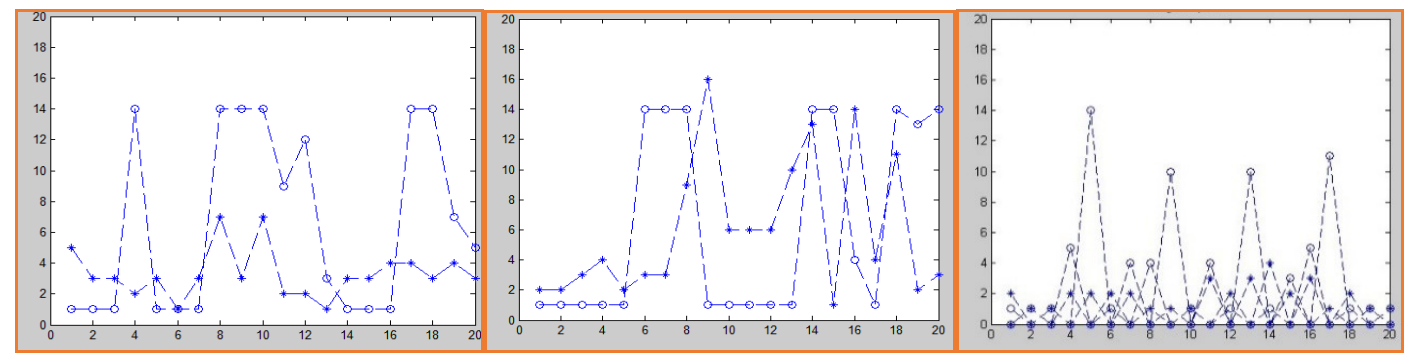

Figure 8. Horizontal, vertical and diagonal profiles of $G^{\mid}$and $L^{\mid}$.

To present the image in the score accurately, two correlations that can measure scores that can measure the relation between structure features are used. They are Spearman rank order correlation coefficient (SRC) and Pearson correlation coefficient (PCC).

The dependencies between features of extracted horizontal, vertical and diagonal profiles can formulate the level of distortion in the image. To find out them, spearman and Pearson correlations between $\mathrm{Pg}, \mathrm{Pl}$ and $\mathrm{Cg}, \mathrm{Cl}$ are calculated. We propose four models Pearson correlation coefficient between Pg and Pl (PRCP), Pearson correlation coefficient between $\mathrm{Cg}$ and $\mathrm{Cl}$ (PRCQ), Spearman rank correlation between Pg and Pl (SRCP), Spearman rank correlation between $\mathrm{Cg}$ and $\mathrm{Cl}$ (SRCQ). The scores of four models and their comparison with Structural similarity value are tabulated in columns below. Highlighted values represent right ordered values in coincidence with level of distortion.

Scores of AWGN distorted images and their relevant SSIM values are recorded in table 1. For Blur, Pearson correlations of conditional probabilities give more equivalence. The scores of blur distorted images and their relevant SSIM values are given in table 2. For AWGN, Spearman correlations of marginal distributions give more equivalence. The scores of images distorted with flicker noise and their relevant SSIM values are recorded in table 3. For flicker noise affected images, Pearson correlations of marginal distributions and conditional probabilities give more equivalence. The scores of JPEG distorted images and their relevant SSIM value are recorded in table 4. For JPEG, Pearson correlations of marginal probabilities give more equivalence. The scores of JPEG2k distorted images and their relevant SSIM values are shown in table 5. For JPEG2k, Spearman correlations of conditional probabilities and Pearson correlations of marginal distributions give more equivalence. Overall, Pearson correlation coefficients of marginal distributions proves to be exemplary. 
International Journal on Cybernetics \& Informatics (IJCI) Vol. 5, No. 4, August 2016

Table 1. Scores of AWGN distorted images and their relevant SSIM value.

\begin{tabular}{|c|c|c|c|c|c|}
\hline Level of Distortion & PRCP & PRCQ & SRCP & SRCQ & SSIM \\
\hline $\mathbf{1}$ & $\mathbf{0 . 2 3 6 8}$ & $\mathbf{0 . 9 2 4 4}$ & 0.3333 & $\mathbf{0 . 8 1 8 2}$ & 0.9869 \\
\hline $\mathbf{2}$ & $\mathbf{0 . 2 6 8 8}$ & $\mathbf{0 . 9 3 2 4}$ & 0.2001 & $\mathbf{0 . 8 5 4 5}$ & 0.9554 \\
\hline $\mathbf{3}$ & $\mathbf{0 . 2 7 9 1}$ & 0.9239 & 0.2256 & $\mathbf{0 . 8 7 8 8}$ & 0.8853 \\
\hline $\mathbf{4}$ & $\mathbf{0 . 2 8 8 1}$ & $\mathbf{0 . 9 3 4 5}$ & 0.2121 & $\mathbf{0 . 9 1 5 7}$ & 0.7781 \\
\hline $\mathbf{5}$ & 0.2881 & 0.9105 & 0.9761 & $\mathbf{0 . 9 2 5 8}$ & 0.6319 \\
\hline
\end{tabular}

Table 2. Scores of Blur distorted images and their relevant SSIM value.

\begin{tabular}{|c|c|c|c|c|c|}
\hline Level of Distortion & PRCP & PRCQ & SRCP & SRCQ & SSIM \\
\hline $\mathbf{1}$ & $\mathbf{0 . 2 2 9 9}$ & 0.9362 & 0.4061 & $\mathbf{0 . 8 1 8 2}$ & 0.9953 \\
\hline $\mathbf{2}$ & $\mathbf{0 . 2 1 4 9}$ & $\mathbf{0 . 9 2 0 6}$ & 0.4061 & $\mathbf{0 . 8 3 0 3}$ & 0.9827 \\
\hline $\mathbf{3}$ & $\mathbf{0 . 2 0 4 5}$ & $\mathbf{0 . 9 3 0 2}$ & $\mathbf{0 . 4 0 6 1}$ & 0.7455 & 0.9437 \\
\hline $\mathbf{4}$ & $\mathbf{0 . 1 7 7 5}$ & $\mathbf{0 . 9 3 0 8}$ & $\mathbf{0 . 4 4 9 9}$ & $\mathbf{0 . 8 9 0 9}$ & 0.8366 \\
\hline $\mathbf{5}$ & $\mathbf{0 . 0 9 4 1}$ & 0.8739 & $\mathbf{0 . 6 3 8 3}$ & $\mathbf{0 . 9 0 3 1}$ & 0.6263 \\
\hline
\end{tabular}

Table 3. Scores of Fnoise distorted images and their relevant SSIM value.

\begin{tabular}{|c|c|c|c|c|c|}
\hline Level of Distortion & PRCP & PRCQ & SRCP & SRCQ & SSIM \\
\hline $\mathbf{1}$ & $\mathbf{0 . 2 5 7 7}$ & $\mathbf{0 . 9 4 6 4}$ & 0.2485 & $\mathbf{0 . 9 0 3 1}$ & 0.9908 \\
\hline $\mathbf{2}$ & $\mathbf{0 . 2 6 8 9}$ & $\mathbf{0 . 9 4 0 7}$ & $\mathbf{0 . 2 0 0 1}$ & 0.8667 & 0.9667 \\
\hline $\mathbf{3}$ & $\mathbf{0 . 2 7 8 1}$ & $\mathbf{0 . 9 3 2 1}$ & 0.2485 & $\mathbf{0 . 8 9 0 9}$ & 0.9162 \\
\hline $\mathbf{4}$ & $\mathbf{0 . 2 8 0 8}$ & $\mathbf{0 . 9 1 7 4}$ & $\mathbf{0 . 2 4 8 5}$ & $\mathbf{0 . 8 7 8 8}$ & 0.8241 \\
\hline $\mathbf{5}$ & $\mathbf{0 . 3 1 6 2}$ & $\mathbf{0 . 8 7 0 3}$ & $\mathbf{0 . 3 0 5 1}$ & $\mathbf{0 . 7 9 3 9}$ & 0.6978 \\
\hline
\end{tabular}

Table 4. Scores of JPEG distorted images and their relevant SSIM value.

\begin{tabular}{|c|c|c|c|c|c|}
\hline Level of Distortion & PRCP & PRCQ & SRCP & SRCQ & SSIM \\
\hline $\mathbf{1}$ & $\mathbf{0 . 2 4 1 2}$ & $\mathbf{0 . 9 2 0 4}$ & 0.3333 & $\mathbf{0 . 8 3 0 5}$ & 0.9912 \\
\hline $\mathbf{2}$ & $\mathbf{0 . 2 5 2 6}$ & 0.8824 & 0.2485 & 0.8909 & 0.9686 \\
\hline $\mathbf{3}$ & $\mathbf{0 . 2 3 7 1}$ & $\mathbf{0 . 9 1 3 3}$ & 0.3708 & $\mathbf{0 . 8 4 2 4}$ & 0.9196 \\
\hline $\mathbf{4}$ & $\mathbf{0 . 1 7 0 6}$ & $\mathbf{0 . 8 9 6 5}$ & 0.3212 & $\mathbf{0 . 8 5 4 5}$ & 0.7931 \\
\hline $\mathbf{5}$ & $\mathbf{0 . 1 4 9 8}$ & $\mathbf{0 . 8 8 1 6}$ & 0.3697 & $\mathbf{0 . 9 1 4 2}$ & 0.6826 \\
\hline
\end{tabular}


International Journal on Cybernetics \& Informatics (IJCI) Vol. 5, No. 4, August 2016

Table 5. Scores of JPEG2k distorted images and their relevant SSIM value.

\begin{tabular}{|c|c|c|c|c|c|}
\hline Level of Distortion & PRCP & PRCQ & SRCP & SRCQ & SSIM \\
\hline $\mathbf{1}$ & $\mathbf{0 . 2 4 1 4}$ & 0.9134 & $\mathbf{0 . 2 9 1 8}$ & 0.9031 & 0.989 \\
\hline $\mathbf{2}$ & $\mathbf{0 . 2 3 2 1}$ & 0.9064 & $\mathbf{0 . 3 3 3 3}$ & 0.8788 & 0.9637 \\
\hline $\mathbf{3}$ & $\mathbf{0 . 1 8 5 4}$ & 0.8377 & $\mathbf{0 . 3 6 1 7}$ & 0.9031 & 0.9008 \\
\hline $\mathbf{4}$ & $\mathbf{0 . 1 3 3 4}$ & 0.9458 & $\mathbf{0 . 3 8 1 8}$ & 0.8349 & 0.7839 \\
\hline $\mathbf{5}$ & $\mathbf{0 . 0 7 4 1}$ & 0.9203 & $\mathbf{0 . 4 3 9 4}$ & 0.8788 & 0.6097 \\
\hline
\end{tabular}

\section{CONCLUSIONS}

Existing BIQA models are complex and involve either in exquisite decompositions or model learning and support vector regression. Few explicit models unlike the proposed method change the features of the image. Keeping this in concern, an attempt is made to use the correlations between the statistics of the local contrast features. Since these are independent, data of the image is not disturbed. In this paper simple procedures to normalise are used to derive joint statistics with joint adaptive normalisation. Marginal distributions and conditional probabilities and their dependencies led to highly competitive performance. Avoiding the training and learning of the features derived, complexity is reduced. Amongst the four models, Pearson correlation coefficient between Pg and Pl (PRCP) proved to be consistent. However, all the four models have affinity with structural similarity. While Pearson correlation is a linear correlation, Spearman is a rank correlation. Hence results are different for different types of distortions in proposed four models with two correlations because of the variation in structural profiles. This proves that when variation in the image structure can define the type of distortion present in the image.This can lead to development of newer models which can determine the type of distortion.

\section{REFERENCES}

[1] Xue and A. C. Bovik, "Blind image quality assessment by joint statistics of gradient magnitude and laplacian of gaussian", IEEE Trans on image processing, 2014

[2] Z. Wang and A. C. Bovik, "Mean squared error: Love it or leave it? A new look at Signal Fidelity Measures", IEEE Trans Signal processing, vol. 26, no. 1, doi.10.1109/MSP.2008.930649

[3] Z. Wang and A. C. Bovik, H. R. Sheikh and E. P. Simoncelli, "Image quality assessment: From error visibility to structural similarity", IEEE Trans. Image Processing, vol. 13, no. 4, pp. 600-612, 2004

[4] H. R. Sheikh, M. F. Sabir and A. C. Bovik, "A Statistical Evaluation of Recent Full Reference Image Quality Assessment Algorithms", IEEE Transactions on Image Processing, vol. 15, no. 11 pp. 3440 3451

[5] A. K. Moorthy and A. C. Bovik, "A two-step framework for constructing blind image quality indices",IEEE Signal Process. Lett. vol. 17, no. 5, pp. 513-516, 2010

[6] M. A. Saad, A.C. Bovik and C. Charrier, "A DCT statistics-based blind image quality index", IEEE Signal Process. Lett. vol. 17, no. 6, pp. 583-586, 2010

[7] X. Marichal, W.Y. Ma and H. Zhang, "Blur determination in the compressed domain using DCT information", Proc. ICIP, pp. 386-390

[8] A. Mittal, A. K. Moorthy and A. C. Bovik, "No-reference image quality assessment in the spatial domain", IEEE Trans. Image Process., vol. 21, no. 12, pp. 4695-4708, 2012

[9] J. Canny, "A computational approach to edge detection", IEEE Trans. Pattern Anal. Mach. Intell., vol. PAMI-8, no. 6, pp. 679-698, 1986

[10] M. A. Saad, A. C. Bovik and C. Charrier, "Blind image quality assessment: A natural scene statistics approach in the DCT domain", IEEE Trans. Image Process., vol. 21, no. 8, pp. 3339-3352, 2012 
International Journal on Cybernetics \& Informatics (IJCI) Vol. 5, No. 4, August 2016

[11] A. K. Moorthy and A. C. Bovik, "Blind image quality assessment: From natural scene statistics to perceptual quality", IEEE Trans. Image Process., vol. 20, no. 12, pp. 3350-3364, 2011

[12] D. Marr and E. Hildreth, "Theory of edge detection", Proc. Roy. Soc. London B, Biol. Sci., vol. 207, no. 1167 , pp. $187-217,1980$

[13] G.-H. Chen, C.-L. Yang and S.-L. Xie, "Gradient-based structural similarity for image quality assessment", Proc. ICIP, pp. 2929-2932

[14] H. R. Sheikh, Z. Wang, L. Cormack and A. C. Bovik, Live Image Quality Assessment Database Release 2., 2011, [online] Available: online 